limiting magnitude in the mixtures with just less than the critical chlorine pressure. Further, while the explosion was more usually apparently instantaneous on admitting the light, there was sometimes a delay of about one second in mixtures close to the limit, when a Draper effect of similar magnitude was observable.

A more detailed account of this reaction and a theory of the kinetic mechanism will appear at an early date.

University Chemical Laboratory, R. G. W. NorrisH. Cambridge, April 29.

\section{Photochemical Interactions of Hydrogen with Chlorine and Bromine.}

THE photochemical interaction of chlorine and hydrogen has been recently described as an inexhaustible theme. Despite the fact that it has been the subject of so many carefully performed experimental researches, it is not yet possible to describe the stages of transformation of the two gaseous elements into the compound hydrogen chloride. We have recently solved one of the outstanding difficulties. The rate of union of the corresponding reaction between hydrogen and bromine is nearly proportional to the square root of the intensity of the light, that is, to the light absorbed by the bromine in unit time, whereas determinations of the rate of union of chlorine and hydrogen have shown it to be almost directly proportional to the intensity of the light.

This marked difference in behaviour we have shown to be due to the fact that the determinations of the rates of reaction with hydrogen and bromine have been made in the absence of impurities which inhibit the reaction, whilst those with hydrogen and chlorine have been made with mixtures which contain such impurities, notably oxygen. Our recent experiments show that if the chlorine used in the experiment is separated from the inhibitive impurity, oxygen, by fractional distillation, and then afterwards purified from other inhibitive impurities by washing it with pure water, the rate of reaction in question is, like that of the reaction between hydrogen and bromine, no longer directly proportional to the intensity of the light, but more nearly to its square root. Moreover, it was shown in this laboratory by E. R. Boller, almost two years ago, that if a mixture of hydrogen and bromine contains a very small quantity of nitric oxide - an impurity which had previously been shown to be a powerful inhibitor-the rate of formation of hydrogen bromide became proportional to the intensity of the light. Therefore, so far as the influence of the light is concerned, these two analogous photochemical changes show the expected correspondence.

Leoline Jenkins Laboratories, D. L. Chapman. F. B. GibBs, Jesus College, Oxford, May 9.

Optical Activity dependent on Co-ordinated Nickel.

Is continuation of our researches on co-ordination compounds containing $a a^{\prime}$-dipyridyl (dipy) we have prepared the following pink complex nickelous salts : the chloride [ $\mathrm{Ni} 3$ dipy] $\mathrm{Cl}_{2}, 6 \mathrm{H}_{2} \mathrm{O}$; the corresponding hexahydrated bromide, iodide, and nitrate, and the thiocyanate [Ni 3dipy] $(\mathrm{CNS})_{2}, 3 \mathrm{H}_{2} \mathrm{O}$.

From the chloride and ammonium $d$-tartrate was obtained a readily soluble dextrorotatory tartrate, $[\mathrm{Ni} 3 \operatorname{dipy}] \mathrm{C}_{4} \mathrm{H}_{4} \mathrm{O}_{6}, 6 \mathrm{H}_{2} \mathrm{O}$, which on double decomposition with ammonium chloride furnished an optically active tris- $a a^{\prime}$-dipyridyl nickelous chloride. When viewed in the polarimeter illuminated by a mercury are using the yellow line, a 0.5 per cent aqueous solution of this chloride in a 2 d.cm. tube gave a maximum rotation of $+5 \cdot 5^{\circ}$ which at $20^{\circ}$ decreased to $+0.08^{\circ}$ in one hour. The rate of diminution of optical activity between these limits was that required for a unimolecular reaction. The maximum activity corresponds with a specific rotation $[a]+550^{\circ}$ and with a molecular rotation $[M]+3877^{\circ}$.

Analogous complex salts have been obtained with bivalent manganese and similar cobaltous compounds are under examination.

The $a a^{\prime}$-dipyridyl required for these experiments was obtained by Hein and Retter's method of dehydrogenating pyridine with ferric chloride under pressure, and in this preparation we have so far isolated some ten additional products, including a tripyridyl (tripy) which gives rise to well-defined coordination compounds with bivalent iron and nickel of the general type [M 2tripy] $\mathbf{X}_{2}$.

G. T. Morgan.

F. H. Burstall.

Chemical Research Laboratory,

Teddington, Middlesex, May 16.

\section{Emission Bands in the Mercury Spectrum under Low Excitation.}

Is a letter published under the above title in NATURE of May 2, it was stated that the series of bands which appear in absorption of the unexcited vapour in the region from $\lambda 2943$ to $\lambda 2614$ could be obtained in fluorescent emission by exciting with the iron are in the region on the long wave side of the resonance line $\lambda 2537$. It is not necessary to excite close to the resonance line in order to get them. This band series accordingly forms part of what I have called the 'wing effect'.

A further very definite result is now to be communicated. The 'core effect', that is, the fluorescence obtained when stimulation is by the exact wave-length of the atomic resonance line as given by a cooled mercury lamp, also includes a band series in the same region of the spectrum, but it is not identical with, or, so far as can be seen at present, definitely related to, the previous band series forming part of the wing effect.

It is identical with the series which I formerly measured in electrically stimulated vapour. ${ }^{1}$ I then called it the emission series of the less refrangible region, to distinguish it from the other series in the same region which had only been obtained in absorption of the unexcited vapour. These names are no longer distinctive, and I shall speak for the present, and quite provisionally, of the core series and the wing series, according to the mode of excitation in fluorescence.

The wing series is related to the apparently continuous maximum around $\lambda 3300$ at its tail end by the fact that both are greatly enhanced by superheating the vapour.

The core series, on the other hand, seems to be related to the apparently continuous maximum around 2650 at its head, which latter forms part of the core effect, but not of the wing effect. Neither the core series nor the maximum 2650 at its head are at all enhanced by superheating.

These various relations come out very strongly in the photographs, which it is hoped to reproduce, and which will make them clearer than a short written account can be expected to do.

Terling Place,

Chelmsford, Essex, May 25.

1 Proc. Roy. Soc., A, vol. 116, p. $703 ; 1927$.

No. 3214, VoL. 127] 